\title{
Processo de desenvolvimento de software na visão da engenharia simultânea e dos ambientes cooperativos
}

\section{Resumo}

$\mathrm{O}$ artigo mostra o potencial e as possibilidades do desenvolvimento de software em ambientes cooperativos, que sejam apoiados na engenharia simultânea (CE - Concurrent Engineering), bem como os problemas e limitações atuais. São discutidos os conceitos básicos para a efetivação de um ambiente deste tipo e as principais tecnologias empregadas.

\section{Abstract}

The present article shows the potential and the possibilities of the software developing in cooperative ambiances, which be supportes by Concurrent Engineering as well as the problems and its real limitations. Here are discussed the basic concepts for the achievement of a typical ambiance ant the principal technologies employed on it.

\section{1 - Introdução}

No desenvolvimento de software, o crescimento da complexidade aliada às restrições de tempo e de custos levam, cada vez mais, à necessidade de uma maior estruturação no gerenciamento de projetos. Prazos dilatados, especificações não levantadas de modo adequado, funcionamento incorreto do software, e outros problemas, contribuem para o mito de que produzir software é diferente de outras atividades humanas.

Com a finalidade de melhorar este quadro, práticas consideradas ruins são eliminadas, impondo-se métodos formais na especificação e no projeto dos softwares, bem como esperam-se ganhos de produtividade e qualidade, adotandose o uso de softwares do tipo CASE (Computer Aided Software Engineering).

$\mathrm{O}$ ambiente de desenvolvimento de software, cada vez mais complexo, utiliza tempos de desenvolvimento muito longos e manipula orçamentos altos, cuja estrutura envolve muitos processos, equipes, sistemas e ferramentas. Neste tipo de projetos, os principais problemas estão localizados nas inconsistências das informações do próprio projeto, implicando no aumento do tempo e do custo de desenvolvimento.

Uma forma de minimizar estes problemas, tornando esses projetos mais produtivos e gerenciáveis, está na utilização da engenharia simultânea apoiando a metodologia de desenvolvimento, onde muitas das atividades do ciclo de vida são realizadas de modo simultâneo. O problema maior neste caso é o conveniente gerenciamento dos processos simultâneos e das informações envolvidas. Para isso, o artigo propõe a utilização da Engenharia Simultânea (CE - Current Engineering) junto com o Processo Unificado, cada um complementando o outro para o desenvolvimento de melhores sistemas.

*Doutor e Mestre pelo Departamento de Engenharia de Computação e Sistemas Digitais da Escola Politécnica da Universidade de São Paulo. Engenheiro Eletrônico pela Universidade Mayor de San Marcos, (Lima-Peru). Professor do Departamento de Engenharia de Computação e Sistemas Digitais da Escola Politécnica da Universidade de São Paulo.

**Doutorando em Engenharia de Software pela Escola Politécnica da USP. Mestre em Administração. Engenheiro Eletrônico. Professor da FITO - Fundação Instituto Tecnológico de Osasco. Professor das Faculdades Integradas "Campos Salles". 


\section{2- Métodos cooperativos de desenvolvimento de software}

\subsection{Engenharia simultânea}

Para o desenvolvimento de sistemas, a engenharia de software clássica pode se beneficiar de ferramentas já consagradas em campos como o da engenharia de hardware. Uma dessas ferramentas é a engenharia simultânea (ES), também conhecida como engenharia concorrente (CE - Concurrent Engineering) ou paralela.

Pode-se definir a ES como uma técnica de gerenciamento para reduzir o ciclo de entrega ao mercado dentro do desenvolvimento de produtos, através da execução simultânea de atividades e do processamento de informações do projeto desse produto. Embora utilizada com sucesso no desenvolvimento não seqüencial de hardware, ainda não aparece no desenvolvimento completo de software, ocorrendo em partes isoladas do projeto, como codificação e testes.

Conforme Hartley, a diferença entre produtos que utilizam engenharia convencional e simultânea, é sentida principalmente no tempo de projeto e nos custos associados com as mudanças ao longo do projeto, como apresentado na figura 1, que ilustra as diferenças da utilização da ES dentro do ciclo de desenvolvimento de hardware.

Um próximo passo dentro da indústria de software, segundo Blackman et al., seria o casamento entre a engenharia de software e a ES, o que deve levar a uma engenharia simultânea de software (ESS).

\section{Figura 1 - Comparação do desenvolvimento de produtos (hardware) utilizando a engenharia convencional e a simultânea ${ }^{1}$}

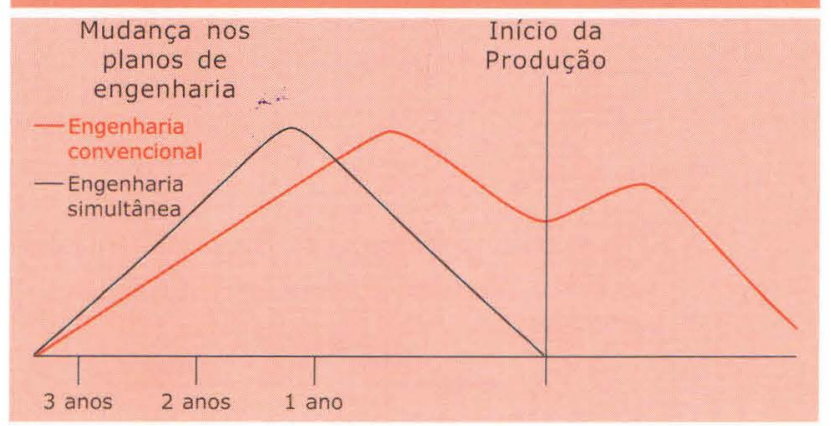

Não existe uma definição comum para a engenharia simultânea e a confusão se dá, principalmente no projeto e no desenvolvimento onde a concorrência aparece de duas formas distintas:

a. Concorrência de atividades - refere-se às tarefas e atividades de projeto executadas simultaneamente por diferentes pessoas ou grupos. A concorrência de atividades pode ser hierarquizada do seguinte modo:

a.1. Sobreposição dentro de um estágio - é a mais simples, e ocorre quando o problema relativo ao projeto é subdividido em módulos desenvolvidos em paralelo.

a.2. Sobreposição entre estágios - envolvendo atividades concorrentes entre diversos estágios do processo de desenvolvimento como, por exemplo, o trabalho concorrente do detalhamento do projeto e das atividades de teste.

a.3. Sobreposição de hardware e de softwareocorre em ambientes onde deve haver uma integração entre hardware e software, e onde mudanças de hardware implicam em mudanças de software, e vice-versa.

a.4. Conjunção entre projetos - apresenta um novo desafio, o de que componentes projetados para um produto podem ser reutilizados em novas versões desse ou de outro produto.

b. Concorrência da informação - refere-se à forma integrada das diversas equipes envolvidas em um desenvolvimento, onde tudo o que diz respeito a diferentes funções - cliente, $\mathrm{P} \& \mathrm{D}$, projeto, engenharia, manufatura, vendas e serviços - é acessado através de um fluxo compartilhado de informações. O fluxo de informações para suportar este tipo de concorrência pode se dar de 3 formas:

b.1. Informações da linha de frente - que devem ser consideradas no início do projeto e dizem respeito às atividades fim, como engenharia de processos, manufatura, serviços ao consumidor e até desenvolvimento do mercado futuro. Estas informações dão um primeiro alerta sobre questões que, se não consideradas, podem gerar custos com reprojeto e retrabalhos mais tarde. A correta utilização destas informações pode auxiliar na criação de projetos mais robustos e que podem ser reutlizados.

'Baseado em Hartley, John R. Engenharia Simultânea, pp. 98-101 
b.2. Informações para início de trabalho - dizem respeito à transferência da informação preliminar, de base, fluindo a partir das atividades iniciais do projeto para todas as equipes envolvidas.

b.3. Informações em dois sentidos comunicação rica e intensa entre as equipes, enquanto executam atividades concorrentes. $\mathrm{O}$ fluxo de informação inclui comunicações sobre potenciais soluções de projeto e de mudanças, o que evita a inviabilidade do projeto e os problemas de interface. Difere das duas anteriores, já que as anteriores são fluxos unidirecionais de informação. Aqui as informações vão de uma função para outra, como, por exemplo, na sobreposição de hardware e de software, onde se apresenta uma situação de um fluxo bidirecional de informações crítico, refinando os elementos progressivamente, evitando-se problemas futuros de integração.

Neste contexto apresentado, a tarefa básica do gerente de projetos é a de administrar as complexas relações entre o fluxo de informações e as atividades concorrentes. Para que se estabeleça a relação entre a concorrência de atividades e de informações, necessita-se acrescentar dois conceitos básicos:

a. Modularidade na arquitetura - que diz respeito à divisão dos problemas do projeto em módulos com funcionalidades e interfaces bem definidas. $\mathrm{O}$ modo pelo qual esta divisão é feita determina o tipo e o nível do fluxo de informações necessário para suportar uma atividade concorrente.

b. Sincronismo - que diz respeito à coordenação de atividades paralelas. Precisa ser uma função do líder do projeto, que deve assegurar que os grupos trabalhem com comunicação paralela um com o outro, sincronizando prazos e garantindo uma integração efetiva.

Pequenos processamentos de lotes (batch) facilitam todas as formas de concorrência de informações. A transferênciâ freqüente de informações em pequenas quantidades, ao invés do modo periódico e em grandes lotes, suporta a atividade concorrente, reduzindo o intervalo entre as comunicações.

\subsection{CSCW e a engenharia simultânea}

Antes de entrar propriamente na engenharia simultânea de software, é conveniente estudar-se o trabalho cooperativo suportado por computador, que apresenta uma série de princípios que serão largamente utilizados pela engenharia simultânea aplicada ao desenvolvimento de software.

Segundo Blair \& Rodden, as pesquisas sobre o trabalho cooperativo suportado por computador (CSCW - Computer Supported Cooperative Work) iniciaram-se em 1984, com vistas a discutir o desenvolvimento de sistemas de computador que possam suportar equipes multidisciplinares agregadas em atividades de trabalho. A emergência da CSCW resulta dos efeitos combinados do desenvolvimento tecnológico e mudanças na natureza social do trabalho. O CSCW pode ser caracterizado através das seguintes práticas:

a. práticas dinâmicas de trabalho que se baseiam mais no contexto corrente e no nível de atividade do trabalho, e não necessariamente em normas ou procedimentos. Além disso, tarefas diferentes podem permitir interfaces, serem renegociadas e revistas dentro do progresso do trabalho;

b. modos de interação, síncronos ou assíncronos, que podem, na prática, ser comutados de um para outro rapidamente;

c. arranjo dos membros da equipe que podem estar em um mesmo ambiente ou, remotamente, localizados. Neste último caso, necessita-se de uma infraestrutura de comunicações, que pode variar desde uma rede multi-serviço de alta velocidade, através de comunicações via Internet, até redes sem fio de baixa velocidade;

d. tipos de mídia que, utilizadas pelas aplicações de trabalho em grupo, podem variar desde textos e gráficos simples, até animações, voz e vídeo;

e. heterogeneidade de plataformas é uma das constantes dentro de ambientes de trabalho em grupo, como, por exemplo, em projetos com hardwares e sistemas operacionais distintos.

Existem três aspectos do trabalho cooperativo que devem ser considerados em resposta à diversidade de detalhes que o permeiam. São eles:

a. Projeto do CSCW, que diz respeito ao suporte e gerenciamento dos meios pelos quais a aplicação cooperativa será desenvolvida, considerandose o entendimento do caráter social da organização do trabalho. Isto implica dizer que duas mentalidades distintas, uma técnica e outra social, de- 
vem conviver durante o desenvolvimento do projeto, utilizando um vocabulário comum, bem como para a incorporação de uma consistência cultural que deverá se traduzir em um ganho de informação para o trabalho cooperativo.

b. Serviços genéricos de CSCW devem prover sustentação para aplicações cooperativas. Alguns potenciais serviços podem ser os de mecanismos de percepção, de concorrência, de acesso e de localização, que devem prover o senso de ação que existe em um trabalho cooperativo. Os serviços de interface devem prover facilidades e arquiteturas, para coordenar e gerenciar interações entre as diversas camadas de usuários e os serviços de informação compartilhada são geralmente os primeiros meios usados para desenvolver um entendimento compartilhado, através de vários usuários trabalhando em conjunto. Os elementos para este compartilhamento de informações estão descritos na Tabela I.

c. Infra-estrutura, cuja discussão centra-se em três tópicos chamados de tecnologias núcleo de sistemas distribuídos, com ênfase em: sistemas abertos, provendo acesso transparente a serviços, a partir de qualquer lugar, em ambientes distribuídos e heterogêneos; suporte à mobilidade, utilizando as tecnologias de comunicação atualmente disponíveis, e suporte a multimídia e Internet, onde cada usuário pode requerer diferentes quantidades de serviços, dependendo de suas aplicações.

\section{Proposta para desenvolvimento de} software utilizando a engenharia simultânea

\subsection{Engenharia simultânea no} desenvolvimento de software

Conforme citação de Becerra (1997), definese a engenharia simultânea como o "método sistemático aplicado ao desenvolvimento integrado dos serviços ou produtos, enfatizando a importância da satisfação dos requisitos do usuário, incluindo formas de cooperação, responsabilidade e compartilhamento nas equipes, de forma que as decisões de projeto sejam produto de um processo paralelo, sincronizados pelo eficiente sistema de informação, até produzir um consenso".

Neste paralelismo, pode-se, por exemplo, estar

Tabela 1 - Elementos de um serviço de informações compartilhado

\begin{tabular}{|l|l|}
\hline \multicolumn{1}{|c|}{ Elementos de Serviço } & \multicolumn{1}{c|}{ Descrição } \\
\hline Travamento & $\begin{array}{l}\text { Um conjunto de facilidades para suportar a coordenação de acessos } \\
\text { concorrentes compartilhando objetos entre os usuários }\end{array}$ \\
\hline Controle de Versão & $\begin{array}{l}\text { Facilidades de suporte e gerenciamento de criação e de manutenção } \\
\text { de versões em ambientes cooperativos }\end{array}$ \\
\hline Histórico & $\begin{array}{l}\text { Conjunto de mecanismos históricos que promovem a cooperação sín- } \\
\text { crona através do histórico dos objetos e seu uso }\end{array}$ \\
\hline Acesso & $\begin{array}{l}\text { Facilidades que controlam a habilidade do usuário em acessar dife- } \\
\text { rentes aspectos de um serviço }\end{array}$ \\
\hline Argumentações e visões & $\begin{array}{l}\text { Facilidades que permitem a definição de diferentes visões de objetos } \\
\text { dentro de um serviço e o uso dos mesmos }\end{array}$ \\
\hline Eventos, Filtragens e Subscrição & $\begin{array}{l}\text { Conjunto de serviços que permitem a manipulação e o gerenciamen- } \\
\text { to de eventos de um serviço, que permite diferentes focos de inte- } \\
\text { resse, a serem definidos por diferentes grupos de usuários }\end{array}$ \\
\hline Percepção & $\begin{array}{l}\text { Um conjunto de facilidades que permite aos objetos gerirem o nível de } \\
\text { percepção que eles podem fornecer aos diferentes usuários do serviço }\end{array}$ \\
\hline $\begin{array}{l}\text { Interação e apresentação } \\
\text { em aplicações cooperativas }\end{array}$ & $\begin{array}{l}\text { A apresentação de diferentes objetos e aspectos dos serviços para } \\
\text { usuários finais }\end{array}$ \\
\hline Referências Externas & $\begin{array}{l}\text { Facilidades proporcionadas por serviços de objetos compartilhados } \\
\text { que permitem alguma forma de ligação entre objetos de um serviço e } \\
\text { objetos de informação fora do serviço }\end{array}$ \\
\hline
\end{tabular}


testando um requerimento que não é mais válido, ou que tenha sido modificado. A solução para isso são mecanismos paralelos automatizados, sincronizados entre si.

São dois os elementos importantes na estrutura do ambiente de projeto: o ciclo de vida paralelo de desenvolvimento e a equipe de desenvolvimento que, por sua vez, possuem as seguintes características:

- execução das fases do projeto são realizadas de forma paralela, existindo um trabalho cooperativo entre todas as fases;

- informações de projeto são armazenadas numa base de dados única, e disponibilizadas da mesma forma para todos os membros das equipes, incluindo as gerências;

- gerência não exclusiva de cada fase, adotando-se uma gerência geral, onde as atividades de coordenação são fatores críticos;

- sistema de informação com características tais que permitam a interoperabilidade de todos os seus elementos, suportando um ambiente cooperativo para a manipulação eficiente da informação de projeto por todos os elementos dentro da empresa.

Um outro elemento importante, neste tipo de ambiente de projeto, é a equipe de desenvolvimento multidisciplinar, constituída de elementos com diferentes perfis profissionais trabalhando de modo cooperativo. Esta equipe está, na verdade, composta de muitas equipes que, por sua vez, estão constituídas de muitos profissionais de diferentes áreas, distribuídos fisicamente na corporação e integrados através do sistema de informação, compondo o que se chama de equipe virtual, funcionando como um elemento único e coerente. Para que isso seja possível, a integração e a coerência da equipe virtual devem ser suportadas por um Banco de Dados que seja único dentro do projeto.

\subsection{Banco de dados de suporte ao desenvolvimento}

Uma forma de minimizar os riscos de erros no projeto é através da definição de um modelo conceitual de dados, denominado de base de dados única de projeto. Esta base é uma estrutura abstrata de dados, representando as complexidades de dados referentes ao projeto, estando disponível a todos os membros da equipe virtual.

Deve-se notar que a base de dados serve todo o projeto e toda a corporação, permitindo que todos tenham acesso aos mesmos dados, que se tornam, desta forma, únicos e consistentes. A estrutura lógica de uma base de dados única de projeto está composta dos seguintes tipos de informação:

- Informações referentes ao produto: informações técnicas dos produtos e serviços desenvolvidos pela empresa, que podem ser: especificações dos requisitos, definições de projeto, diferentes modelos, diversas arquiteturas, especificações técnicas sobre tecnologia, considerações de segurança e planos de testes.

- Informações referentes ao processo: definições dos processos que são aplicados no desenvolvimento dos produtos ou serviços, como fluxos de trabalhos (workflows), estados do processo, requisitos de desenvolvimento, definição de atividades e funções, regras de interação, formas de coordenação, controle de versões e usuários envolvidos.

- Informações referentes à empresa: informações referentes à estrutura de suporte do desenvolvimento, como recursos humanos, ferramentas de apoio ao projeto, planejamentos, planos estratégicos, normas corporativas, documentos de decisões, distribuição de trabalho, históricos de produtos e serviços.

Essa base de dados única de projeto é interligada às bases setoriais que, por sua vez, estão ligadas às bases locais. Em cada nível de interação existe um mecanismo de controle de acesso e versão para manter a consistência dessas informações.

De modo hierárquico, as bases setoriais possuem uma cópia parcial das informações da base única, assim como as bases locais possuem cópias parciais da base setorial. As bases locais e setoriais são utilizadas nas atividades específicas de cada grupo de usuários. Existem blocos de controle de acesso e versões das diferentes bases, que realizam cópias das informações atualizadas, de modo automático ou segundo a necessidade do cliente, executando as funções de coordenação entre as diversas bases, evitando a inconsistência dos dados.

Uma possível arquitetura $^{2}$ para um sistema de

${ }^{2}$ Baseada em Melo, p. 26 
cooperação e concorrência, apresentada na figura 2, deve ser composta por:

- Um gerenciador de recursos - base de dados para armazenar objetos e atividades, e acompanhar o progresso do projeto. Este banco suporta um modelo de dados entidade-relacionamento, que pode ser estendida com conceitos de orientação a objeto, como herança, métodos e encapsulamento.

- Um gerenciador de atividades - responsável pelo controle da integração. Este gerenciador é guiado por regras do tipo temporal-evento-condição-ação (TECA - Temporal-Event-ConditionAction), e suportado em parte por mecanismos de gatilho (trigger) do Banco de Dados. Os mecanismos de gatilho foram habilitados a manipular expressões temporais.

- Um gerenciador de processos - oferece os conceitos do tipo de processo e tarefa. Este gerenciador é responsável pela comunicação e o sincronismo entre as equipes e entre os agentes envolvidos no projeto, controlando também a consistência de objetos complexos usados simultaneamente em diferentes ambientes de trabalho por diferentes agentes.

A implementação do gerenciador de recursos, de atividades e de processos, através da proposta de uma base de dados única, pode ser descrita, conforme o formalismo conceitual e físico tradicional.

\subsection{A ESS no processo unificado}

A partir de diversas considerações de autores ligados ao chamado processo unificado de desenvolvimento, pode-se defini-lo como uma abordagem que, com disciplina, captura o essencial de muitas das melhores práticas de desenvolvimento de software, permitindo a determinação e o gerenciamento de tarefas e responsabilidades. Seu objetivo é a criação de software de alta qualidade, dentro do tempo e dos orçamentos pré-determinados, e que atendam as necessidades dos usuários.

Pode-se definir o processo unificado, a partir de quatro visões distintas: (a) ele é um processo dentro da engenharia de software, pois permite que se utilize uma abordagem disciplinada para determinar tarefas e responsabilidades; (b) é um produto, quando se considera o uso do Rational Unified Process, uma ferramenta de desenvolvimento de software da Rational Software; e (c) é um framework de um processo que pode ser adaptado e estendido para atender uma organização em particular.

Cabe lembrar que, para a integração com a engenharia simultânea, é importante o modo preferencial de trabalho do processo unificado. Nele, o processo seqüencial (cascata) é substituído por um mais dinâmico, que é o interativo. Deste modo, pode-se obter um produto muito melhor, conduzindo pequenos processos simultâneos gerenciados de modo adequado, para que

\section{Figura 2 - Arquitetura conceitual de um possivel sistema de trabalho cooperativo e simultâneo}

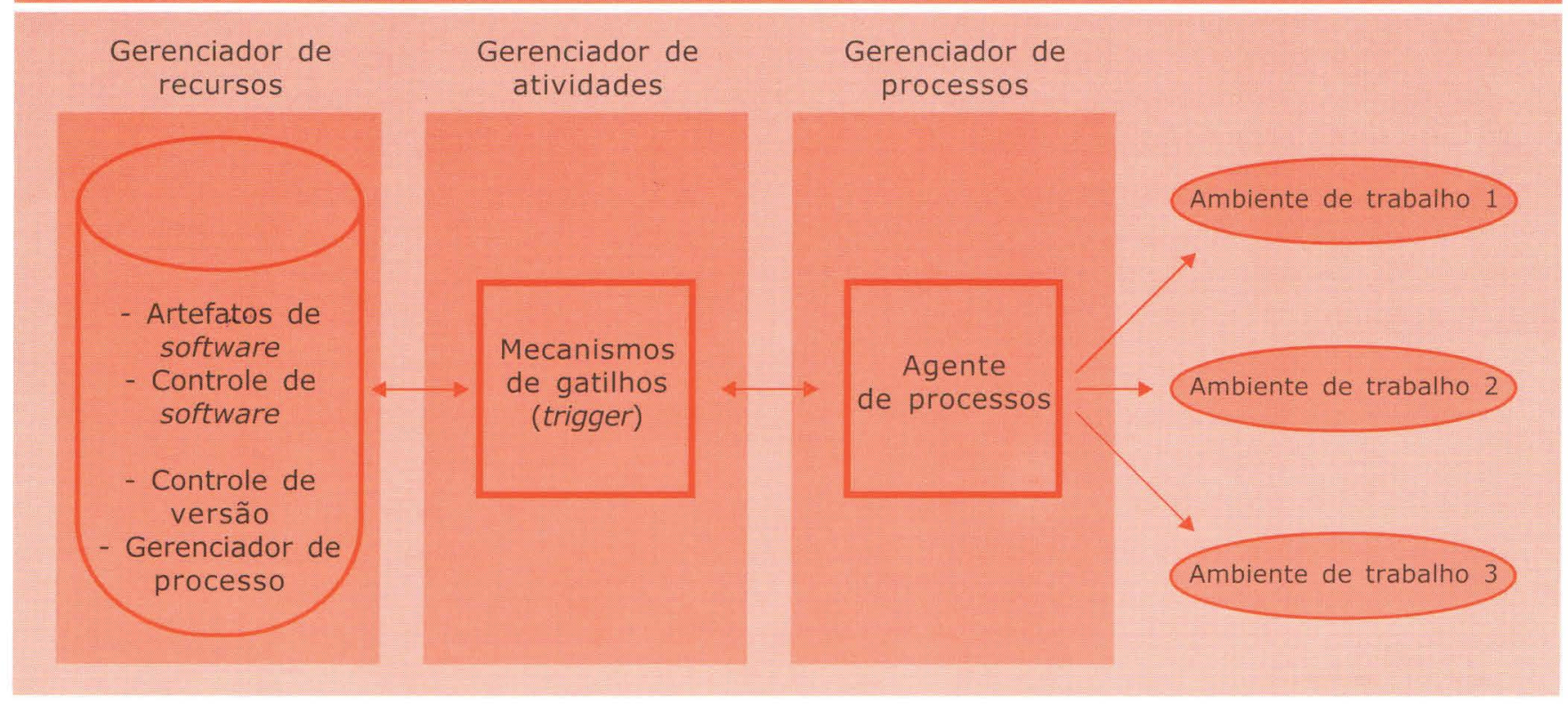




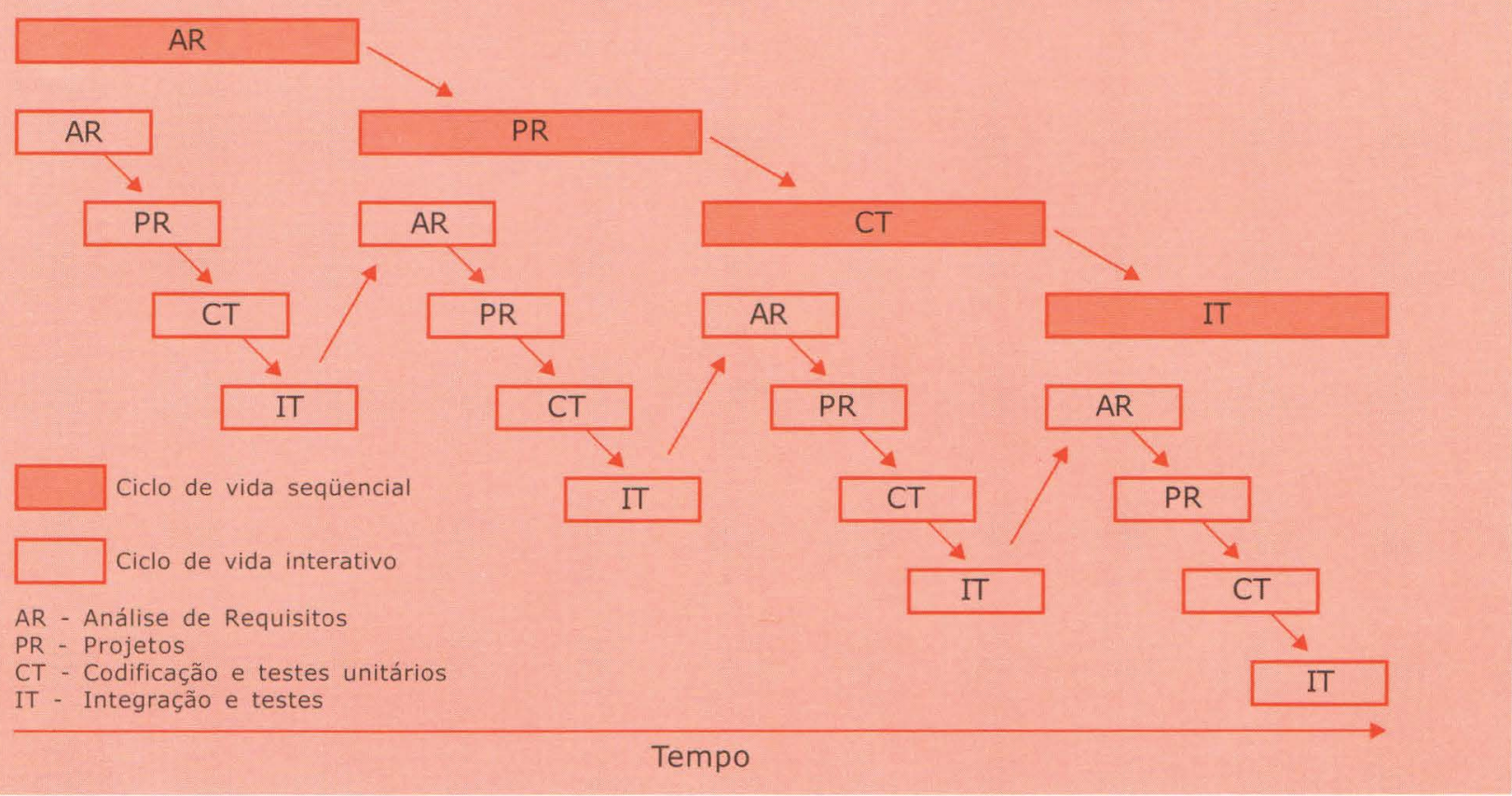

se possa obter um produto consistente.

Assim, conforme apresentação na figura 3, o tradicional processo seqüencial pode dar lugar a um processo interativo onde, além das superposições, todo o processo está contido em cada um dos processos, compondo um ambiente recursivo. A conjugação da engenharia simultânea de software pode se dar justamente através desse ciclo de vida interativo. Os mecanismos, para que se possa determinar a relação entre o processo unificado e a engenharia simultânea, devem ocorrer nesta região, onde a interatividade e a recursividade necessitam de elementos de gerenciamento que possam controlar o processo.

Para apresentar esta proposta de junção da engenharia simultânea com o processo unificado, a figura 4 mostra o trabalho de desenvolvimento de software através de 4 níveis distintos, que estão, no entanto, interligados e interagentes. Estes níveis são:

- Nível 1 - Gerência Simultânea - Encarregado do gerenciamento de todas as atividades do processo unificado e suas concorrências. Seu Banco de Dados de Projeto, funcionando como um gerenciador de recursos, armazena e controla informações sobre os artefatos e processos de software, bem como informações de controle de versão e de progresso, através da realimentação conveniente de informações decorrentes das ações do nível 3. Dentro deste nível, deve-se adotar um recurso gerenciador de atividades que funciona como um gatilho para ativar as atividades que sejam necessárias em dado momento. Finalmente, um gerenciador de processos funciona como um elemento sincronizador das atividades envolvidas, garantindo sua ativação ou desativação em momentos convenientes.

- Nível 2 - Composto de Bases de Dados setoriais ou locais que possuem cópia dos elementos que lhes são necessários para a execução do processo, disponibilizando-as para o usuário no momento adequado. $O$ controle dessas Bases de Dados locais deve ficar a cargo de um gerenciador maior (no nível 1) que coordena a concorrência entre as atividades evitando conflitos.

- Nível 3 - São as atividades interativas, acontecendo de modo interativo, mas sob a supervisão do nível 2, que por sua vez é supervisionado pelo nível 1. Deste modo, tem-se o controle de quais atividades estão sendo executadas e o status de cada uma, além das informações devidas a outras atividades. Através de um processo de realimentação, este nível informa o nível 1 , que providencia a execução ou bloqueio de uma ação.

- Nível 4 - Do produto ou serviço, alterado constantemente pelo nível 3 que o realimenta com informações de status e providências necessárias para 
Nível 1 - Gerência Simultânea

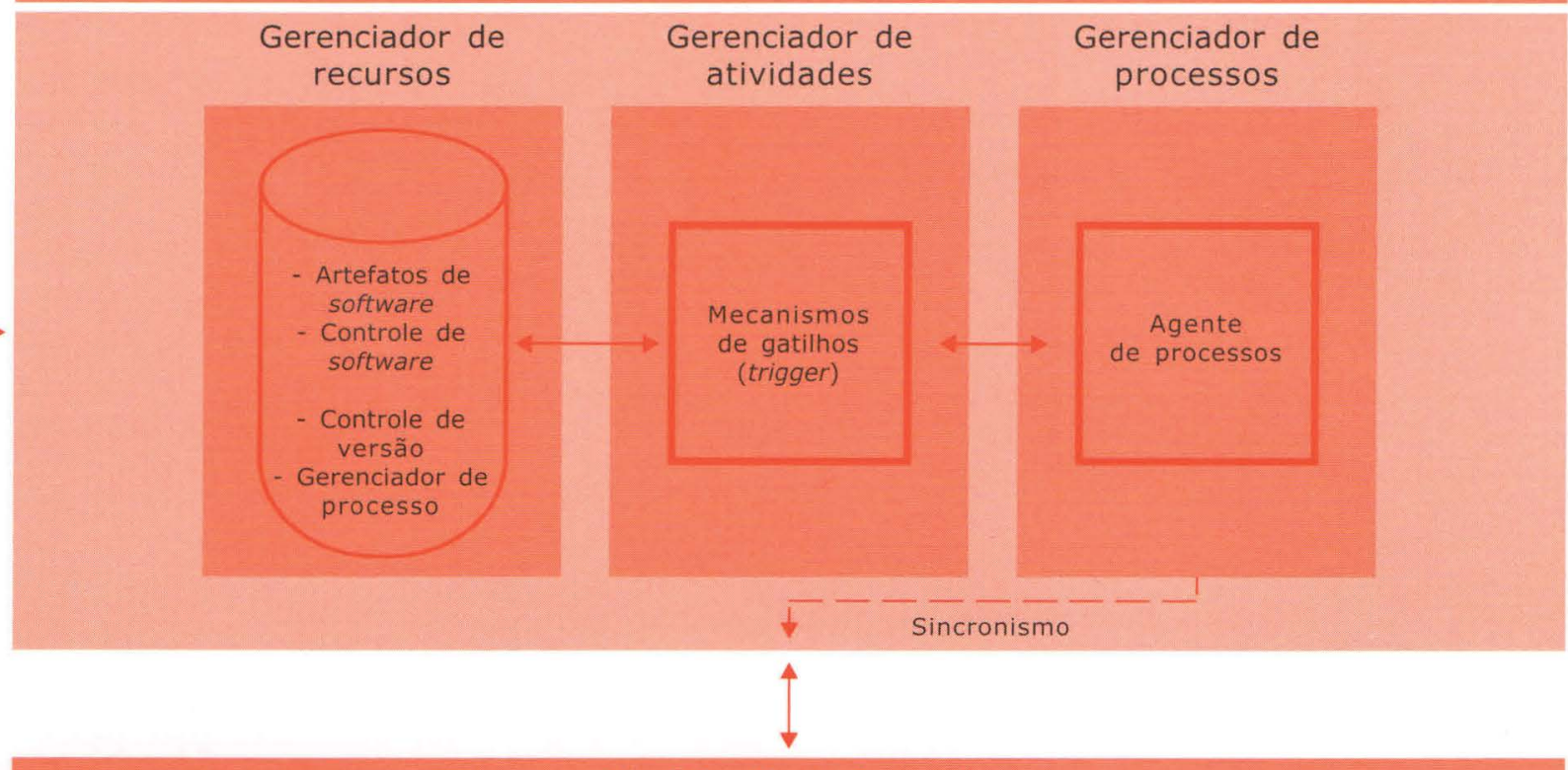

Nível 2 - Controle Local

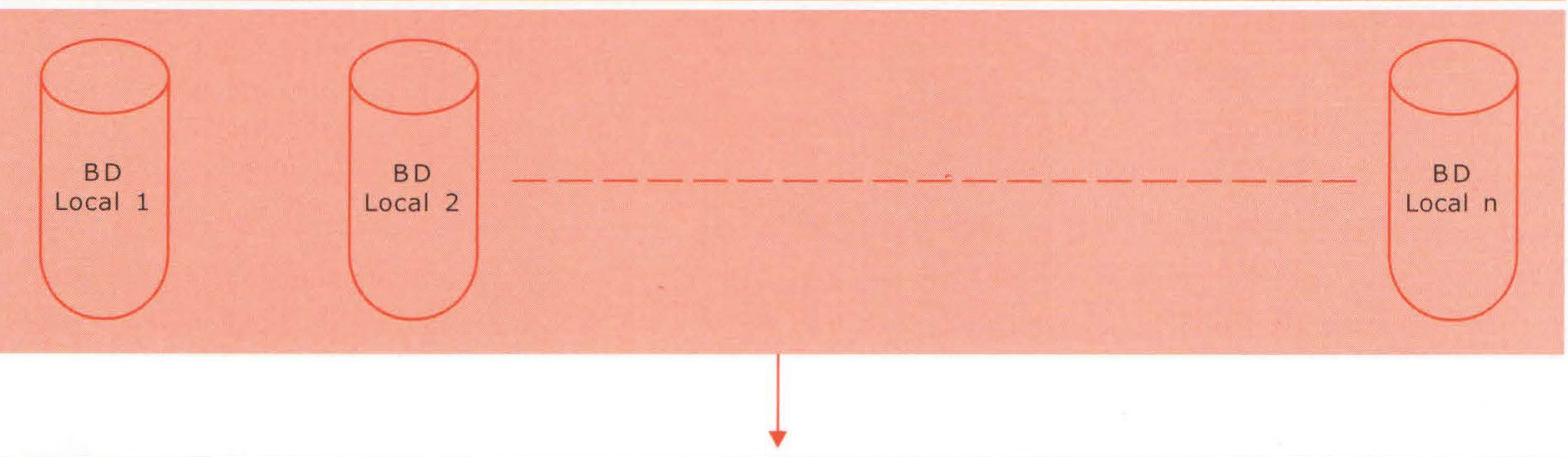

Nível 3 - Atividades (Processo Unificado - Interativo)

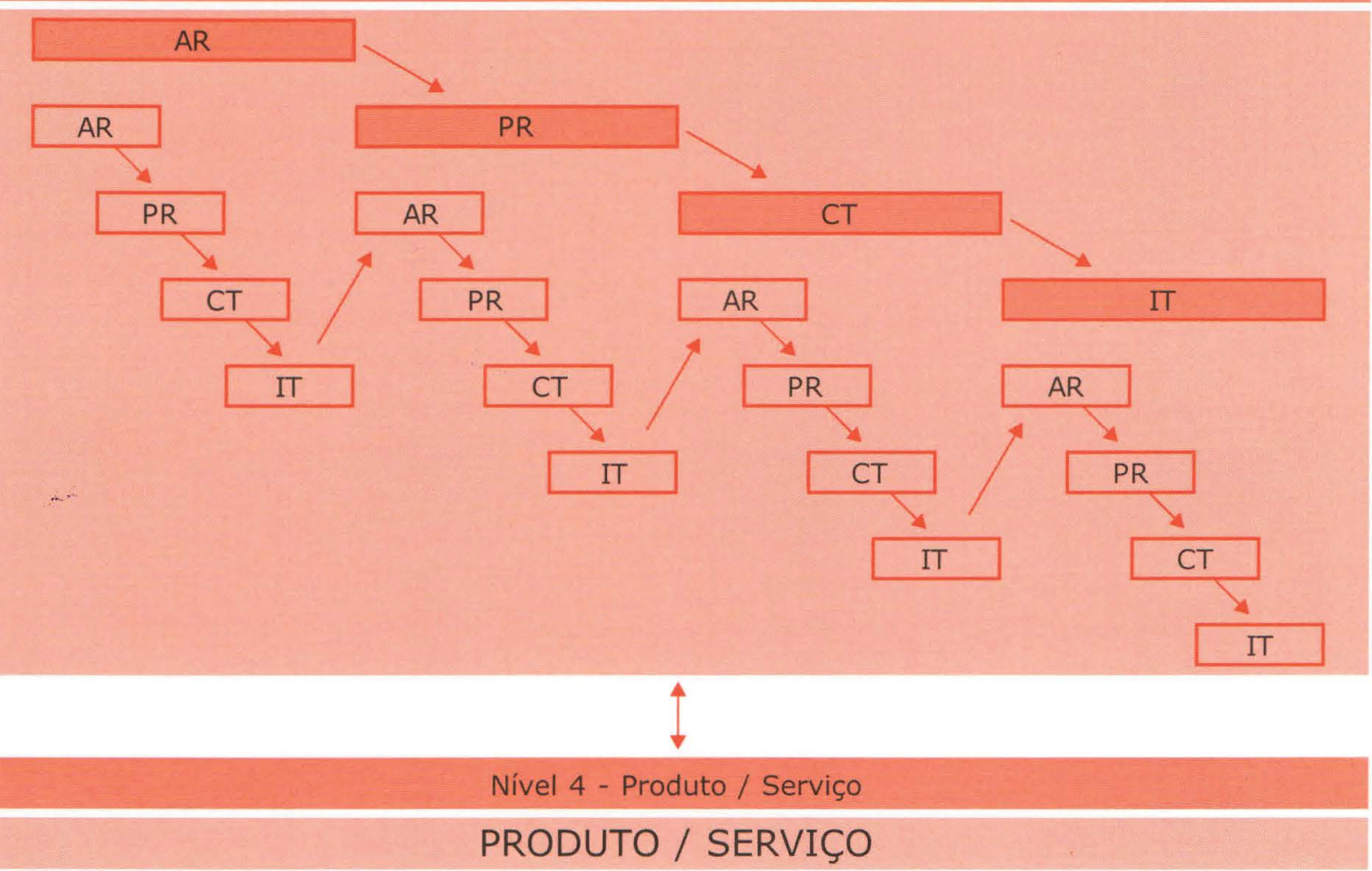


eventuais correções e/ou mudanças necessárias. Estas informações realimentarão o nível 1 que pode acionar ou bloquear uma ou mais atividades.

Este primeiro modelo integra a engenharia simultânea de software (ESS) dentro do processo unificado, permitindo que se aproveite o potencial de desenvolvimento de software de ambas.

\section{Conclusão}

Embora ainda existam muitas questões sobre como a engenharia simultânea pode auxiliar no desenvolvimento de software, parece que a utilização do processo unificado para a produção de software, apoiado pela engenharia simultânea, desencadeará uma série de estudos que estabeleçam uma ligação mais forte entre ambas.

Tudo indica que um bom caminho para se iniciar a busca da engenharia simultânea de software (ESS) seja o uso do processo unificado, que apresenta uma abertura maior e ênfase mais atual dentro dos trabalhos interativos. Esta interatividade entre processos e pessoas envolvidas parece necessitar daquilo que a engenharia simultânea pode oferecer: o gerenciamento de atividades múltiplas através do compartilhamento de informações.

O modelo de níveis apresentado neste trabalho, é um ponto de partida para a conjunção do processo unificado com a engenharia simultânea. Novos estudos deverão indicar ampliações e/ou alterações neste modelo, e uma possível utilização da estrutura em duas dimensões do processo unificado.

\section{REFERÊNCIAS BIBLIOGRÁFICAS}

BASTER, Greg; KONANA, Prabhudev; SCOTT, Judy E. Business components: a case study of bankers truste Australia limited. Communications of the ACM, v. 44, nº 5, p. 92-98, May 2001.

BECERRA, J.L.R. Aplicação do padrão ODP no desenvolvimento de sistemas distribuídos e abertos aplicados em automação. São Paulo, 1997. Tese (Doutorado) - Escola Politécnica, Universidade de São Paulo.

. Especificação, projeto e implementação de um controlador de célula flexível aplicado na manufatura. São Paulo, 1993.

Dissertação (Mestrado) - Escola Politécnica, Universidade de São Paulo.

BLACKBURN, Joseph D.; HOEDEMAKER, Geert; WASSENHOVE, Luk N. Van. Concurrent software engineering: prospects and pitfalls. IEEE Transactions on Engineering Management, v. 43, n. 2, p. 179-188, May 1996.

BLAIR, Gordon S.; RODDEN, Tom. The opportunities and challenges of CSCW. JournaI of the Brazilian Computer Society, v. 1, n. 1, p. 3-14, June 1994.

BOEHM, Barry. Unifying software engineering and systems engineering. IEEE Computer, p. 114-116, Mar. 2000.

BOOCH, Grady. Object oriented design - whit applications. Redwood City, CA, The Benjamin/Cummings Publishing Company, 1991. BRAVO, Marco. Orientação a objetos: parte da solução para gerência e produção. Developers'CIO Magazine, v. 4, n. 43, p. 14-16, Mar. 2000.

FURLAN, José David. Modelagem de objetos através da UML - Análise e desenho orientados a objeto. São Paulo : Makron Books, 1998.

HADDAD, Carol J. Operationalizing the concept of concurrent engineering: a case study from the U.S. auto industry. IEEE Transactions on Engineering Management, v. 43, n. 2, p. 124-132, May 1996.

HARTLEY, John R. Engenharia simultânea. Porto Alegre: Bookman, 1992.

HATCH, Melaine; BADINELLI, Ralph D. A concurrent optimization methodology for concurrent engineering. IEEE Transactions on Engineering Management, v. 46, n. 1, p.72-86, Feb. 1999.

HAUPTMAN, Oscar; HIRJI, Karim K. The influence of process concurrency on project outcomes in product development: an empirical study of cross-functional teams. IEEE Transactions on Engineering Management, v. 43, n. 2, p. 153-164, May 1996.

HULL, Frank M.; COLLINS, Paul D.; LIKER, Jeffrey K. Composite forms of organization as a strategy for concurrent engineering effectiveness. IEEE Transactions on Engineering Management, v. 43, n. 2, p. 133-142, May 1996.

KING, Nelson; MAJCHRZAK, Ann. Concurrent engineering tools: are the humam issues being ignored?. IEEE Transactions on Engineering Management, v. 43, n. 2, p. 189-201, May 1996.

KORSON, T.;MCGREGOR, D. J. Understating object-oriented: a unifyng paradigm. Commmunications of ACM, v. 33, n. 9, p. 40-60, Sept.1990.

KRUCHTEN, Philippe. The rational unified process: an introdution. Massachusetts,: Addison Wesley Lonman, 2000. 
MELO, Walcélio L. Collaborating software engineering processés in tempo. JournaI of the Brazilian Computer Society, v. 1, n. 1, p. 24-35, July 1994.

NADLER, Gerald. Systems methodology and design. IEEE Transactions on Systems, Man, and Cybernetics, v. SMC-15, n. 6, p. 685 - 697, Nov./Dec. 1985.

NEHMER, J.; MATTERN, F. Framework for the organization of cooperative services in distributed client-server systems. Computer Communications, v. 15, n. 4, p. 261-77, 1992.

PRESSMAN, Roger S. Software engineering - a practitioner's approach . 5.ed. McGraw-Hill Higher Education, Makron Books, 2001. . Engenharia de Software. São Paulo: Makron Books, 1995.

RAFII, Farshad; PERKINS, Sam. Intermationalizing software whit concurrent engineering. IEEE Software, Sept. 1995.

ROSENBLATT, Alfred; WATSON, George F. Concurrent engineering. IEEE Spectrum, pp. 22-37, July 1991.

RUMBAUGH, James et al., Object-oriented modeling and design. New York: Prentice-Hall International Editions, 1991.

STIEMERLING, Oliver; CREMERS, Armin B. The use of cooperation scenarios in the design and evaluation of a CSCW system. IEEE Transactions on Software Engineering, v. 24, n. 12, p. 1171-1181, Dec. 1998.

WHEELER, Tom. O manual de sistemas abertos. Rio de Janeiro: Campus, 1994. 